\title{
Clinical Characteristics and Treatment Response in Microscopic Colitis Based on Age at Diagnosis: A Multicenter Retrospective Study
}

\author{
Amrit K. Kamboj ${ }^{1}$ - Jessica McGoldrick ${ }^{2}$ - Eli Voth ${ }^{3} \cdot$ Daniel Penrice $^{3} \cdot$ June Tome $^{3} \cdot$ Amandeep Gujral $^{2} \cdot$ Kaia Miller $^{2}$. \\ Kristin E. Burke ${ }^{2,4}$. Darrell S. Pardi ${ }^{1}$. Hamed Khalili ${ }^{2,4,5}$ (D)
}

Received: 13 April 2021 / Accepted: 6 July 2021 / Published online: 20 July 2021

(c) The Author(s) 2021

\begin{abstract}
Background Microscopic colitis (MC) primarily affects older adults; thus, data in younger patients are scarce. Aims To compare clinical characteristics and treatment response by age at diagnosis.

Methods This retrospective cohort study was performed at Mayo Clinic and Massachusetts General Hospital. Patients were chosen consecutively using established databases. Patients were 'younger' if age at diagnosis was $\leq 50$ years and 'older' if age $>50$ years. Treatment outcomes were captured for induction (12 \pm 4 weeks), based on the total number of daily stools, and defined as remission (complete resolution), response ( $\geq 50 \%$ improvement), non-response ( $<50 \%$ improvement), and intolerance. Patients were considered 'responders' if they had remission or response and 'non-responders' if they had nonresponse or intolerance.

Results We included 295 patients (52 younger, 243 older). There were no differences in sex, race, MC subtype, and diarrhea severity between groups (all $P>0.05$ ). Younger patients were more likely to have celiac disease $(17.3 \%$ vs. 5.8\%, $P=0.01)$, while older patients had higher BMI (mean 25.0 vs. $\left.23.8 \mathrm{~kg} / \mathrm{m}^{2}, P=0.04\right)$ were more likely smokers $(53.9 \%$ vs. $34.6 \%$, $P=0.01)$ and use NSAIDs $(48.6 \%$ vs. $15.4 \%, P<0.01)$ and statins $(22.6 \%$ vs. $3.8 \%, P<0.01)$. Overall treatment response was highest for budesonide $(88.3 \%)$ and did not differ when comparing older to younger patients $(90.6 \%$ vs. $77.8 \%, P=0.12)$ or by MC subtype (LC, $81.5 \%$ vs. CC, $92.9 \%, P=0.07$ ).

Conclusions There are no significant differences in MC treatment response based on age or disease subtype. These findings support treating patients with MC based on symptom severity rather than age.
\end{abstract}

Keywords Bismuth subsalicylate $\cdot$ Budesonide $\cdot$ Diarrhea $\cdot$ Collagenous colitis $\cdot$ Loperamide $\cdot$ Lymphocytic colitis $\cdot$ Mesalamine $\cdot$ Microscopic colitis

Hamed Khalili

hkalili@mgh.harvard.edu

1 Division of Gastroenterology and Hepatology, Mayo Clinic, Rochester, MN, USA

2 Division of Gastroenterology and Hepatology, Massachusetts General Hospital, Boston, MA, USA

3 Department of Internal Medicine, Mayo Clinic, Rochester, MN, USA

4 Clinical and Translational Epidemiology Unit, Mongan Institute, Massachusetts General Hospital, Boston, MA, USA

5 Cardiovascular and Nutritional Epidemiology Unit, Karolinska Institutet, Stockholm, Sweden

\section{Introduction}

Microscopic colitis (MC) is an inflammatory disease of the large intestine characterized by histologic changes of intraepithelial lymphocytosis and dense inflammatory infiltrate in the lamina propria, with or without expansion of the subepithelial collagen band and a normal or nearly normal endoscopic appearance [1-3]. There are two main subtypes of MC, namely collagenous colitis (CC) and lymphocytic colitis (LC), distinguished by the presence or absence of collagen thickening, respectively. MC is a common cause of chronic diarrhea and fecal incontinence in older adults, accounting for up to $20 \%$ of cases in one study [4]. Epidemiologic studies have shown that the peak age of diagnosis is between the ages of 65 and 75 years with women making up $70 \%$ of cases [5-7]. However, nearly 1 out of 4 cases are 
diagnosed in adults younger than 50 years [5, 8]. Additionally, there have been a few case reports of MC in children $[9,10]$. Nevertheless, little is known about differences in clinical characteristics and treatment response based on age at diagnosis.

The etiology of MC is unknown but thought to be related to aberrant immune response to environment factors, perhaps mediated through perturbances in the gut microbiota. Exposure to certain medications and the presence of other immune-mediated diseases such as celiac disease are among the most well-studied risk factors $[1,2]$. However, the distribution of these potential risk factors varies widely across age suggesting that there may be differences in disease pathophysiology and natural history according to age. In support of this, one recent study showed that patients younger than age 60 years had significantly higher rates of clinical relapse following response to budesonide, highlighting potential differences in disease course according to age of diagnosis [11].

We therefore sought to compare clinical characteristics and treatment response of younger versus older MC patients using already established $\mathrm{MC}$ registries in two large health care systems in the US. A secondary aim was to assess treatment response based on MC subtype.

\section{Methods}

\section{Study Population}

This multicenter retrospective cohort study was performed at Mayo Clinic, Rochester, MN and Massachusetts General Hospital (MGH), Boston, MA. Patients with MC were chosen in consecutive fashion using already characterized cohorts from each institution (Mayo Clinic, 2007-2008; MGH, 2011-2013) [12-14]. We have previously reported our methods for identifying MC cases [14, 15]. Briefly, at Mayo Clinic, MC cases were identified by reviewing pathology reports and confirmed by chart review. At MGH, MC cases were identified from Research Patient Data Registry using a combination of ICD code and text search followed by confirmation through review of pathology reports. Patients with a diagnosis of LC, CC, or incomplete MC were included. At both institutions, patients with a diagnosis of non-specific colitis and those under the age of 18 years were excluded. The biopsy protocols at both institutions were similar and involved taking random biopsies throughout the colon placed in one bottle. At both centers, all MC pathology specimens included had previously been reviewed by an expert gastrointestinal pathologist.

\section{Clinical Characteristics}

At each center, we retrospectively collected detailed information on patient demographics using chart review. Data were collected on age at diagnosis, sex, race, and relevant clinical variables such as body mass index (BMI), smoking status (current, former, never, or unknown), baseline number of bowel movements, histologic subtype (CC or LC), presence or absence of celiac disease at the time of MC diagnosis, and use of medications associated with risk of MC including selective serotonin reuptake inhibitors (SSRIs), proton pump inhibitors (PPIs), non-steroidal anti-inflammatory drugs (NSAIDs), statins, antibiotics, and histamine H2-receptor antagonists at the time of diagnosis. We categorized patients into younger-onset MC (age at diagnosis $\leq 50$ years) and older MC (age of diagnosis $>50$ years). As the median age of diagnosis of MC has been reported to range between 60-65 years in the US and Europe [5-7, 14], we considered age below this cutoff to represent a significantly younger cohort than average.

\section{Treatment Outcomes}

We collected information on all medical treatments received for MC including loperamide, atropine/diphenoxylate, aminosalicylates (5-ASA), bismuth subsalicylate, bile acid sequestrants, antibiotics, budesonide (ileal release and multi-matrix), prednisone, immunomodulators, tumor necrosis factor (TNF) inhibitors, and vedolizumab. We defined treatment as consistent if it was used for at least 8 weeks and patients were excluded if they received less than 8 weeks of therapy. Clinical outcomes were assessed for induction therapy at $12 \pm 4$ weeks after the start of a therapy. Clinical outcomes for maintenance therapy were not captured. For each patient, we included outcomes for all medications used for induction during follow-up. In other words, if different medications were used for induction at different time points during followup, we captured each episode separately and did not limit inclusion to the first episode of induction or to the episode that utilized first-line therapy. Medical treatment used for MC, and not for other causes of diarrhea, was included.

In this study, treatment outcomes were defined a priori and based on changes in the total number of daily bowel movements. More specifically, treatment outcomes were defined as follows: remission (complete resolution), response ( $\geq 50 \%$ improvement), non-response $(<50 \%$ improvement), and intolerance (medication stopped due to side effects). The definition of remission was similar to that proposed by Hjortswang et al., namely $<3$ stools/day and a mean of $<1$ watery stool per day [16]. In patients 
where data on the total number of daily bowel movements were not available, the treatment outcomes were based on the treating physician's note. Patients were considered to be 'responders' if they had remission or response to a treatment and 'non-responders' if they had non-response or intolerance.

\section{Statistical Analysis}

Descriptive variables are reported as median (range) or proportions. We used Fischer's exact test and Wilcoxon ranksum test to compare categorical and continuous variables, respectively. Multivariable logistic regression modeling was used to calculate the odds ratios (OR) and $95 \%$ confidence intervals (CI) while adjusting for celiac disease, smoking history, BMI, and NSAID and statin use. All p values were 2 -sided and $P<0.05$ was considered statistically significant. $\mathrm{R}$ version 3.5.1 was used for all statistical analyses. The study was approved by the institutional review board at Mayo Clinic and Mass General Brigham (MGB).

\section{Results}

We included 295 patients (Mayo $=132, \mathrm{MGH}=163$ ) with median age at diagnosis of 66 years (range 18-94 years). Nearly $76 \%(n=223)$ of cases were female while $97 \%$ $(n=284)$ were white. Table 1 demonstrates baseline characteristics of our participants according to age at diagnosis. While the majority of our cases were diagnosed after the age of 50 years $(n=243,82 \%)$, nearly $18 \%(n=52)$ were diagnosed before age 50 years. The median age of diagnosis among younger and older patients was 39 years (range 18-50 years) and 69 years (range 50-94 years), respectively. In both age groups, there was a similar proportion of LC and CC subtypes. There were also no statistically significant differences in sex, race, and alcohol consumption between the two groups. Younger patients were more likely to have a diagnosis of celiac disease $(17.3 \%$ vs. $5.8 \%, P=0.01)$ while older patients had a higher BMI (25.0 vs. $23.8, P=0.04$ ) and were more likely to be current or former smokers $(53.9 \%$ vs. $34.6 \%, P=0.01)$. Additionally, older patients were more likely to use NSAIDs $(48.6 \%$ vs. $15.4 \%, P<0.01)$ and statins $(22.6 \%$ vs. $3.8 \%, P<0.01)$. The most common SSRIs were citalopram $(n=17,27 \%)$, sertraline $(n=14,22 \%)$, and fluoxetine $(n=13,21 \%)$. The most common PPIs were omeprazole $(n=46,57 \%)$, esomeprazole $(n=14,17 \%)$, and pantoprazole $(n=13$, $16 \%$ ).

There were no differences in baseline symptoms between the two groups, including presence of abdominal pain, weight loss, or number of bowel movements per day. Data on the total number of daily bowel movements were available for $84.7 \%$ of the participants.
Table 1 Baseline characteristics of patients with $\mathrm{MC}$ according to age at diagnosis

\begin{tabular}{|c|c|c|c|c|}
\hline \multirow[t]{2}{*}{ Characteristics } & \multicolumn{4}{|l|}{ Median \pm range or $n(\%)$} \\
\hline & All patients $(n=295)$ & $\begin{array}{l}\text { Age } \leq 50 \text { at the time } \\
\text { of diagnosis }(n=52)\end{array}$ & $\begin{array}{l}\text { Age }>50 \text { at time of } \\
\text { diagnosis }(n=243)\end{array}$ & $P$ value \\
\hline Age & $66(18-94)$ & $38.9(18-50)$ & $69.3(50-94)$ & \\
\hline Sex, female & $223(75.6)$ & $42(80.8)$ & $181(74.5)$ & 0.44 \\
\hline Race, white & $284(96.3)$ & $50(96.1)$ & $234(96.3)$ & 0.94 \\
\hline Body Mass Index $\left(\mathrm{kg} / \mathrm{m}^{2}\right)$ & $24.8(14.8-43.5)$ & $23.8(17.6-36.8)$ & $25.0(14.8-43.5)$ & 0.045 \\
\hline \multicolumn{5}{|l|}{ MC subtype } \\
\hline Lymphocytic colitis & $129(43.7)$ & $28(53.8)$ & $101(41.5)$ & 0.14 \\
\hline Collagenous colitis & $153(51.9)$ & $23(44.2)$ & $130(53.5)$ & 0.29 \\
\hline Incomplete MC & $13(4.4)$ & $1(1.9)$ & $12(4.9)$ & 0.56 \\
\hline Celiac disease* & $23(7.8)$ & $9(17.3)$ & $14(5.8)$ & 0.01 \\
\hline Smoking history & $149(50.5)$ & $18(34.6)$ & $131(53.9)$ & 0.01 \\
\hline Alcohol consumption & $147(49.8)$ & $30(57.7)$ & $117(48.1)$ & 0.42 \\
\hline \multicolumn{5}{|l|}{ Medications** } \\
\hline SSRIs & $63(21.4)$ & $11(21.2)$ & $52(21.4)$ & 1.00 \\
\hline PPIs & $81(27.5)$ & $10(19.2)$ & $71(29.2)$ & 0.16 \\
\hline NSAIDs & $126(42.7)$ & $8(15.4)$ & $118(48.6)$ & $<0.01$ \\
\hline Antibiotics & $20(6.8)$ & $4(7.7)$ & $16(6.6)$ & 1.00 \\
\hline $\mathrm{H} 2$ blockers & $8(2.7)$ & $2(3.8)$ & $6(2.5)$ & 0.95 \\
\hline Statins & $57(19.3)$ & $2(3.8)$ & $55(22.6)$ & $<0.01$ \\
\hline
\end{tabular}

*Celiac disease confirmed by serology and/or biopsy

**Medications used within 30 days of microscopic colitis diagnosis 
In multivariable analysis, there were no differences in presence of celiac disease, history of smoking, and statin use between younger versus older patients after adjusting for differences in baseline characteristics (Table 2). Older patients were more than two times (OR 2.35, 95\% CI 1.08-5.13, $P=0.03)$ more likely to be overweight or obese and more than five times (OR 5.15, 95\% CI 2.02-13.1, $P<0.01$ ) more likely to use NSAIDs compared to younger patients.

A distribution of commonly used MC medications stratified by patient age and overall response rates is summarized in Table 3 . There were no significant differences in treatment response to loperamide, budesonide, mesalamine, and bismuth subsalicylate between younger and older patients after adjusting for baseline differences in BMI and NSAID use (Fig. 1). Supplementary Fig. 1 demonstrates treatment response to less commonly utilized medications according to age at diagnosis (all nonsignificant). Younger patients were more likely to achieve remission with budesonide compared to older patients $(n=13$, $48.1 \%$ vs. $n=37,29.1 \%, P=0.04$ ) (Supplementary Fig. 2). We did not observe any significant differences in treatment response according to MC subtypes, although there was a numerical difference toward a more favorable treatment response in CC with budesonide $(92.9 \%$ vs. $81.5 \%$,
Table 2 Multivariableadjusted comparison of disease determinants according to age at diagnosis

\begin{tabular}{lcccr}
\hline Covariates & $\begin{array}{l}\text { Age } \leq 50 \text { years } \\
(n=52)\end{array}$ & $\begin{array}{l}\text { Age }>50 \text { years } \\
(n=243)\end{array}$ & OR (95\% CI) & $P$ value \\
\hline Celiac disease & $9(17.3)$ & $14(5.8)$ & $0.54(0.17-1.79)$ & 0.32 \\
Ever smoking & $18(34.6)$ & $131(53.9)$ & $1.63(0.76-3.46)$ & 0.21 \\
BMI $^{*}\left(>24.8 \mathrm{~kg} / \mathrm{m}^{2}\right)$ & $12(23.1)$ & $104(42.8)$ & $2.35(1.08-5.13)$ & 0.03 \\
NSAID use $^{\mathbb{I l}}$ & $8(15.4)$ & $118(48.6)$ & $5.15(2.02-13.1)$ & $<0.01$ \\
Statin use & $2(3.8)$ & $55(22.6)$ & $4.06(0.90-18.4)$ & 0.07 \\
\hline
\end{tabular}

*BMI $=$ body mass index

${ }^{\mathbb{I}} \mathrm{NSAID}=$ non-steroidal anti-inflammatory drug

Table 3 Frequency and overall response rate of common MC treatments by age of diagnosis

\begin{tabular}{lcccc}
\hline Common therapies & All patients $(n=295)$ & Age $\leq 50$ years $(n=52)$ & Age $>50$ years $(n=243)$ & $\begin{array}{l}\text { Overall } \\
\text { response rate, } \\
n(\%)\end{array}$ \\
\hline Loperamide & $115(38.9)$ & $16(30.8 \%)$ & $99(40.7 \%)$ & $60(52.2)$ \\
Budesonide & $154(52.2)$ & $27(51.9 \%)$ & $127(52.3 \%)$ & $136(88.3)$ \\
Mesalamine & $54(18.3)$ & $18(34.6 \%)$ & $36(14.8 \%)$ & $25(46.3)$ \\
Bismuth subsalicylate & $117(39.7)$ & $23(44.2 \%)$ & $94(38.7 \%)$ & $73(62.4)$ \\
\hline
\end{tabular}

Fig. 1 Comparison of response to common MC therapies according to age of diagnosis

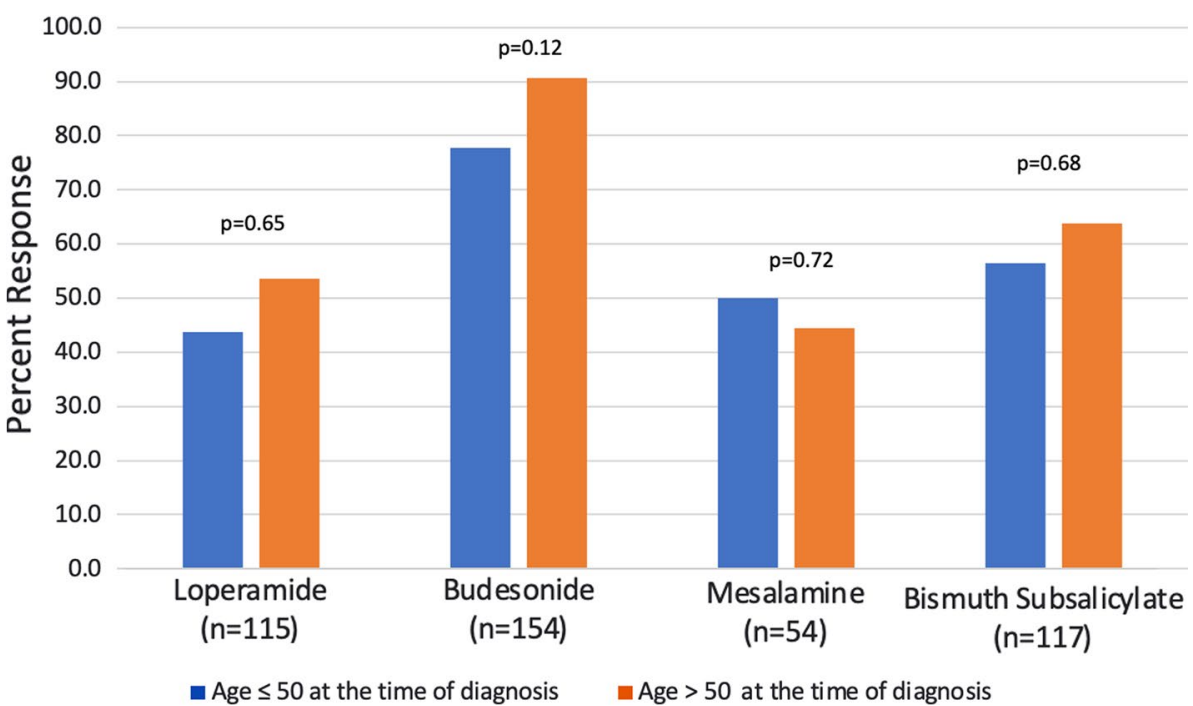


Fig. 2 Comparison of response therapy according to disease subtype

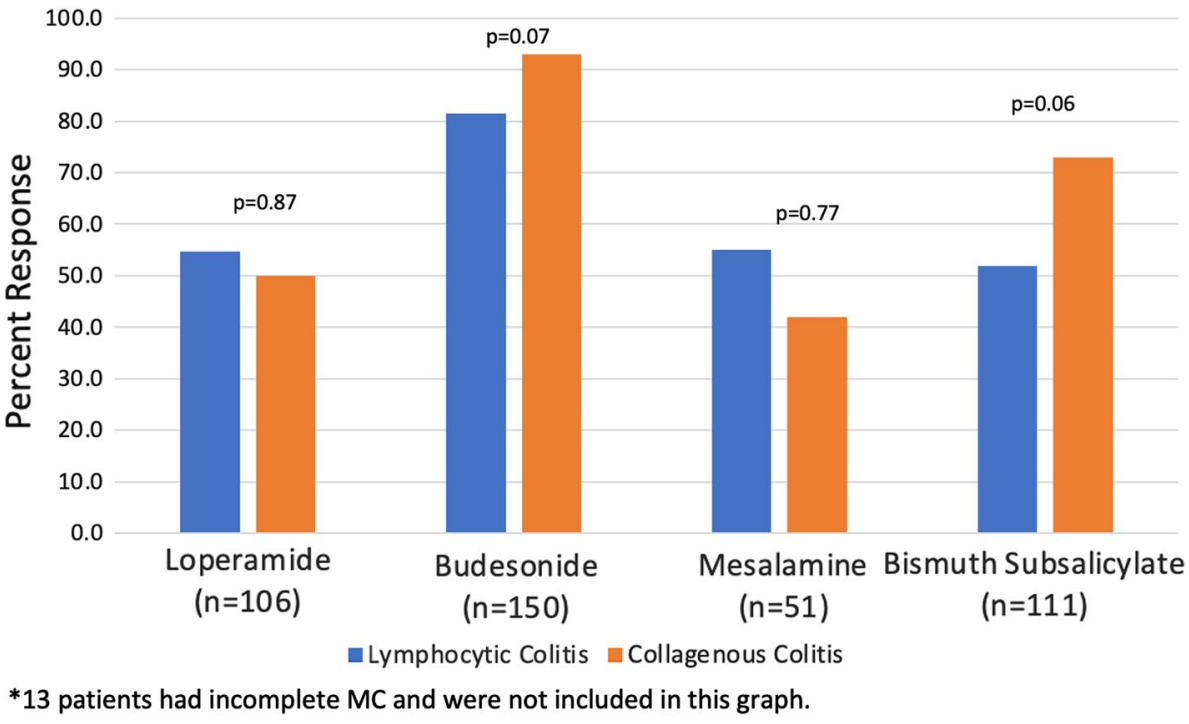

$P=0.07)$ and bismuth subsalicylate $(72.9 \%$ vs. $51.9 \%$, $P=0.06$ ) compared to LC (Fig. 2).

\section{Discussion}

In this multicenter retrospective cohort study, we highlight the clinical characteristics and treatment response to commonly utilized medications in younger $(\leq 50$ years of age) and older ( $>50$ years) patients with MC. To our knowledge, this is the first study to assess the clinically important question of whether treatment outcomes in patients with MC vary according to age. We show that there are no significant differences in treatment response to loperamide, bismuth subsalicylate, budesonide, and mesalamine between younger and older patients with MC. Additionally, we did not observe any significant difference in treatment response according to MC subtype. This study suggests that MC treatment should not be based on patient age or MC subtype.

We found that most baseline characteristics were similar between younger and older patients with MC including sex, race, MC subtype, and gastrointestinal symptoms. Prior studies have shown a similar female predominance regardless of age $[8,17]$. There was a higher proportion of younger patients with celiac disease although this association did not remain significant on multivariate analysis. We demonstrate that older patients with $\mathrm{MC}$ are more than two times more likely to be overweight or obese and more than five times more likely to use NSAIDs compared to younger patients. NSAIDs are commonly used in older adults for pain control (e.g., osteoarthritis) and have been implicated as having a high likelihood of causing MC $[1,18,19]$. A thorough medication review should be performed in all patients with MC, especially the older adults, and discontinuation of implicated drugs should be strongly be considered if safe and feasible.
In some patients with $\mathrm{MC}$, discontinuation of a causative medication can lead to resolution of colitis, or at least make it more responsive to therapy $[18,20]$.

In this study, there were no significant differences in treatment response to loperamide, bismuth subsalicylate, budesonide, and mesalamine in younger compared to older patients. While a prior study found that older age at MC diagnosis was associated with a better response to bismuth subsalicylate (OR 1.76, 95\% CI 1.21-2.56) [14], our study found no such association. Decisions on management of patients with MC should not place an undue emphasis on patient age, albeit medication side effects in the older population should be considered. Rather, treatment should be guided by the severity of patient symptoms with a goal of decreasing symptoms and improving quality of life. Previously, Hjortswang et al. demonstrated that patients with a mean of $\geq 3$ stools or $\geq 1$ watery stool daily had significantly impaired health-related quality of life [16]. Similarly, another study found a significant correlation between six symptoms, namely number of unformed stools daily, presence of nocturnal stools, abdominal pain, weight loss, fecal urgency, and fecal incontinence and quality of life [21]. Therefore, these symptoms can be used by physicians to assess patient's initial symptoms and evaluate response to treatment.

In addition, we demonstrate that there were no significant differences in treatment response in patients with LC compared to $\mathrm{CC}$, consistent with prior studies [14, 22]. Colussi et al. [14] also found no differences in treatment response based on MC subtype for commonly used medications including budesonide, mesalamine, bismuth subsalicylate, and cholestyramine, although this study had a sample size of 131 patients and may have been underpowered to detect such differences. Moreover, a systematic review and meta-analysis found no difference in treatment response between LC and CC with use of shortterm budesonide [22]. While there are characteristic histologic 
differences between LC (increased intraepithelial lymphocytes) and CC (thickened collagen band), these differences do not appear to impact response to treatment, and therefore the two subtypes are considered to represent a spectrum of the same disease and should be treated similarly $[1,23]$.

There are a number of strengths and limitations to this study. This is a multicenter retrospective cohort study with data from two different patient populations (Rochester, MN and Boston, MA) and a robust sample size of 295 patients. While MC is more common in older adults, younger patients accounted for a significant (18\%) proportion of our study population allowing for adequate comparisons between these groups. We also were able to explore treatment response with each of these specific MC subtypes rather than MC as a whole. Moreover, we collected data on several potential confounders including comorbid conditions (celiac disease and history of smoking or alcohol use) and frequently implicated medications.

Despite our rigorous approach, important potential confounders such as data on exogenous hormone use, presence of other immune-mediated conditions, and genetic susceptibility were not collected in our study. While we had a large sample size in comparison to prior studies, there were a limited number of patients in a number of subgroups that make it difficult to adjust for more confounders. Moreover, the study may have been underpowered to detect some differences in smaller subgroups. For example, while not statistically significant, patients with CC in this study had a greater treatment response to budesonide and bismuth subsalicylate compared to those with LC. There was also a numerical difference toward greater response to budesonide in older versus younger patients, which did not reach statistical significance. In contrast, we found that younger patients were more likely to achieve remission with budesonide compared to older patients, although this was based on a small sample size. Adequately powered, prospective studies are needed to further study these differences. Also, future studies should consider exploring factors that may explain differences in treatment response such as the role of immune response (i.e., immunosenescence), gut microbiome, or pharmacologic triggers. Additionally, given that this study was performed at two large tertiary care centers, referral and selection bias may have been present. In this study, $7.8 \%$ of the cohort had celiac disease which is higher than the $2.7 \%$ previously reported in a population-based study [6]. This fact, together with the primarily Caucasian population in our cohort, may limit the generalizability of the results.

\section{Conclusion}

In a retrospective study from two large centers in the US, we observed no significant differences in treatment response to commonly used medications between younger and older patients with MC. In addition, treatment response was similar for patients with LC and CC. These findings suggest that MC treatment should not be based on patient age or MC subtype. While our study compared clinical characteristics and treatment response in MC based on age at diagnosis, it remains to be investigated whether there are differences in the pathogenesis of $\mathrm{MC}$ according to age of diagnosis.

Supplementary Information The online version contains supplementary material available at https://doi.org/10.1007/s10620-021-07162-4.

Acknowledgments DSP has consulted for Otsuka, Janssen, Merck, Casdin Capital, Salix, Nestle, C3 Jian, Seres Therapeutics, Assembly Bioscience, and has received research support from Seres Therapeutics, Atlantic, Takeda, Salix, Merck, and Pfizer. HK has consulted for AbbVie and receives grant funding from Takeda and Pfizer. HK is also funded by American College of Gastroenterology Clinical Research Award, the Crohn's and Colitis Foundation Senior Research Award, the Becker Foundation, and the National Institute of Health (NIH) R01 AG068390.

Funding Open access funding provided by Karolinska Institute.

\section{Declarations}

Conflict of interest The authors have no conflicts of interest to report.

Open Access This article is licensed under a Creative Commons Attribution-NonCommercial 4.0 International License, which permits any non-commercial use, sharing, adaptation, distribution and reproduction in any medium or format, as long as you give appropriate credit to the original author(s) and the source, provide a link to the Creative Commons licence, and indicate if changes were made. The images or other third party material in this article are included in the article's Creative Commons licence, unless indicated otherwise in a credit line to the material. If material is not included in the article's Creative Commons licence and your intended use is not permitted by statutory regulation or exceeds the permitted use, you will need to obtain permission directly from the copyright holder. To view a copy of this licence, visit http://creativecommons.org/licenses/by-nc/4.0/.

\section{References}

1. Pardi DS. Diagnosis and management of microscopic colitis. Am J Gastroenterol 2017;112:78-85.

2. Miehlke S, Verhaegh B, Tontini GE, Madisch A, Langner C, Münch A. Microscopic colitis: pathophysiology and clinical management. Lancet Gastroenterol Hepatol 2019;4:305-314.

3. Zylberberg HM, Kamboj AK, De Cuir N et al. Medication use and microscopic colitis: a multicentre retrospective cohort study. Aliment Pharmacol Ther 2021;53:1209-1215.

4. Bohr J, Wickbom A, Hegedus A, Nyhlin N, Hörnquist EH, Tysk C. Diagnosis and management of microscopic colitis: current perspectives. Clin Exp Gastroenterol 2014;7:273-284.

5. Bergman D, Clements MS, Khalili H, Agréus L, Hultcrantz $\mathrm{R}$, Ludvigsson JF. A nationwide cohort study of the incidence of microscopic colitis in Sweden. Aliment Pharmacol Ther 2019;49:1395-1400.

6. Gentile NM, Khanna S, Loftus EV et al. The epidemiology of microscopic colitis in olmsted county from 2002 to 
2010: a population-based study. Clin Gastroenterol Hepatol 2014;12:838-842.

7. Bonderup OK, Wigh T, Nielsen GL, Pedersen L, Fenger-Gron M. The epidemiology of microscopic colitis: a 10-year pathologybased nationwide Danish cohort study. Scand J Gastroenterol 2015;50:393-398.

8. Bohr J, Tysk C, Eriksson S, Abrahamsson H, Järnerot G. Collagenous colitis: a retrospective study of clinical presentation and treatment in 163 patients. Gut 1996;39:846-851.

9. Camarero C, Leon F, Colino E et al. Collagenous colitis in children: clinicopathologic, microbiologic, and immunologic features. J Pediatr Gastroenterol Nutr 2003;37:508-513.

10. Narla NP, Smyrk TC, Pardi DS, Tung J. Clinical features and treatment responses in pediatric lymphocytic and collagenous colitis. J Pediatr Gastroenterol Nutr 2013;57:557-561.

11. Miehlke S, Madisch A, Voss C et al. Long-term follow-up of collagenous colitis after induction of clinical remission with budesonide. Aliment Pharmacol Ther 2005;22:1115-1119.

12. Kamboj AK, Zylberberg HM, Lane CM et al. Microscopic colitis and risk of colon adenomas: a multicenter retrospective cohort study. Clin Gastroenterol Hepatol. 2021. https://doi.org/10.1016/j. cgh.2021.05.050.

13. Setia N, Alpert L, van der Sloot KW et al. Lymphocytic colitis: pathologic predictors of response to therapy. Hum Pathol 2018;78:1-7.

14. Colussi D, Salari B, Stewart KO et al. Clinical characteristics and patterns and predictors of response to therapy in collagenous and lymphocytic colitis. Scand J Gastroenterol 2015;50:1382-1388.

15. Cotter TG, Kamboj AK, Hicks SB, Tremaine WJ, Loftus EV, Pardi DS. Immune modulator therapy for microscopic colitis in a case series of 73 patients. Aliment Pharmacol Ther 2017;46:169-174.
16. Hjortswang H, Tysk C, Bohr J et al. Defining clinical criteria for clinical remission and disease activity in collagenous colitis. Inflamm Bowel Dis 2009;15:1875-1881.

17. Olesen M, Eriksson S, Bohr J, Järnerot G, Tysk C. Lymphocytic colitis: a retrospective clinical study of 199 Swedish patients. Gut 2004;53:536-541.

18. Riddell RH, Tanaka M, Mazzoleni G. Non-steroid anti-inflammatory drugs as a possible cause of collagenous colitis: a case-control study. Gut 1992;33:683-686.

19. Masclee GMC, Coloma PM, Kuipers EJ, Sturkenboom MCJM. Increased risk of microscopic colitis with use of proton pump inhibitors and non-steroidal anti-inflammatory drugs. Am J Gastroenterol 2015;110:749-759.

20. Lucendo AJ. Drug exposure and the risk of microscopic colitis: a critical update. Drugs R D 2017;17:79-89.

21. Cotter TG, Binder M, Loftus EV et al. Development of a microscopic colitis disease activity index: a prospective cohort study. Gut 2018;67:441-446.

22. Stewart MJ, Seow CH, Storr MA. Prednisolone and budesonide for short- and long-term treatment of microscopic colitis: systematic review and meta-analysis. Clin Gastroenterol Hepatol 2011;9:881-890.

23. Jessurun J, Yardley JH, Lee EL, Vendrell DD, Schiller LR, Fordtran JS. Microscopic and collagenous colitis: Different names for the same condition? Gastroenterology 1986;91:1583-1584.

Publisher's Note Springer Nature remains neutral with regard to jurisdictional claims in published maps and institutional affiliations. 